\title{
Metabolic Effects of Cyclic Parenteral Nutrition Infusion in Adults and Children
}

Nutrition in Clinical Practice Volume 25 Number 3 June $2010 \quad 277-281$ (C) 2010 American Society for Parenteral and Enteral Nutrition 10.1177/0884533610368701 http://ncp.sagepub.com hosted at http://online.sagepub.com

\author{
Stephen Marc Stout, PharmD, MS, BCPS ${ }^{1}$ and \\ M. Petrea Cober, PharmD, BCNSP ${ }^{1,2}$
}

Financial disclosure: none declared.

Background: Cyclic (discontinuous) infusion of parenteral nutrition $(\mathrm{PN})$ presents a clear practical advantage over continuous 24-hour infusion in patients receiving long-term or home PN. It is important for nutrition support clinicians to recognize the metabolic consequences of cyclic infusion. Methods: A literature search was conducted through PubMed (1948 to December 2009) and MEDLINE (1950 to November 2009) using the search term parenteral nutrition plus any of the following: cycling, cycle, cyclic, discontinuous, intermittent, bolus. Prospective clinical studies characterizing the metabolic effects of cyclic PN infusion in humans and their references were reviewed. Results: Twenty-five studies met inclusion criteria and were included in the review. Sample sizes were 4-65 (18 studies examined $\leq 20$ patients, 9 examined $\leq 10$ patients). Eight studies had important design elements that limit interpretability. Cyclic and continuous PN infusions result in similar nitrogen balance and circulating counterregulatory hormone concentrations. Following PN infusion, carbohydrate oxidation decreases, fat oxidation increases, and the respiratory quotient decreases. In acutely ill mechanically ventilated patients, carbon dioxide production increases. Cyclic infusion may stabilize liver function tests in some patients with mild hyperbilirubinemia $(\leq 20 \mathrm{ng} / \mathrm{mL})$ on continuous PN infusion. Abrupt infusion initiation may cause hyperglycemia. Abrupt discontinuation may cause hypoglycemia, predominantly in children younger than 2-3 years old, which may decrease with infusion tapering. Cyclic PN infusion is not associated with increased daily calcium, phosphorus, magnesium, or vitamin D losses. Conclusions: These results indicate a favorable risk-benefit profile of cyclic PN infusion in most patients receiving long-term or home PN, in support of current practices. (Nutr Clin Pract. 2010;25:277-281)

Keywords: parenteral nutrition; home parenteral nutrition; infusion; adults; pediatrics; neonatology
$\mathrm{P}$ arenteral nutrition $(\mathrm{PN})$ is the intravenous provision of nutrients to patients in whom enteral feeding is insufficient or contraindicated. PN may be provided via a central or peripheral vein and may be used as stand-alone nutrition support or as an adjunct to enteral nutrition. In adults, common indications for PN include inflammatory bowel disease, ischemic bowel, radiation enteritis, bowel obstruction, motility disorders, and many others. ${ }^{1}$ In children, common indications include prematurity, necrotizing enterocolitis, pancreatitis, and short bowel syndrome. ${ }^{1}$ Patients who are acutely ill or require short-term $\mathrm{PN}$ typically receive $\mathrm{PN}$ as a 24-hour continuous infusion. In stable patients who are expected to have a longer course of $\mathrm{PN}$ or receive treatment at home, PN is often administered on a cyclic (discontinuous) schedule.

From the ${ }^{1}$ Lexi-Comp, Inc, Hudson, Ohio, and; ${ }^{2}$ University of Michigan Hospitals and Health Centers, Ann Arbor, Michigan.

Address correspondence to: Stephen Marc Stout, Lexi-Comp, Inc, 1100 Terex Rd, Hudson, OH 44236-3771; e-mail smstout@ umich.edu.
Cyclic PN infusion is more convenient than continuous infusion for patients and families. Daily stopping of the infusion temporarily frees patients from infusion equipment for participation in school, work, and social activities. However, PN is needed for nutrition support and is also associated with development of metabolic complications with substantial morbidity and mortality (eg, hepatobiliary dysfunction)..$^{2-4}$ It is therefore important that nutrition support clinicians recognize the metabolic consequences of cyclic infusion so the practical benefits of cyclic infusion may be weighed against the potential risks. This review was done to synthesize the available clinical data describing the metabolic effects of cyclic PN infusion.

\section{Methods}

A literature search was conducted through PubMed (1948 to December 2009) and MEDLINE (1950 to third week of November 2009) using the search term parenteral nutrition plus any of the following: cycling, cycle, cyclic, discontinuous, intermittent, bolus. Relevant 
publications and their references cited were reviewed for inclusion. Original English-language reports of prospective clinical studies characterizing the metabolic effects of cyclic PN infusion in humans were included in the review. This included studies comparing cyclic infusion with continuous infusion, studies comparing the cyclic infusion period with the noninfusion period, and studies investigating the metabolic effects of initiation and discontinuation of infusions. Studies published only in abstract form and studies exclusively investigating continuous sequential infusion methods were not included. No attempt was made to pool study data for analysis. Pooled analysis was not considered based on high interstudy variability in sample selection, study protocol, and data reporting.

\section{Results}

\section{Studies and Level of Evidence}

A total of 25 publications met the specified criteria and were included in the final literature review. Of these, 10 used a randomized parallel-group or crossover design $(\mathrm{n}=5-65), 11$ used a before-after or nonrandomized crossover design $(n=4-48)$, and 4 used a nonrandomized parallel-group design $(\mathrm{n}=8-10)$. None used blinding.

\section{Nutrient Utilization}

Protein metabolism and nitrogen balance. Several small studies have consistently demonstrated similar nitrogen balance achieved with continuous and cyclic PN infusion $(\mathrm{N}=5-14) .^{5-10}$ Comparable nitrogen balance was also achieved in a randomized parallel-group study of 16 traumatized or infected mechanically ventilated patients. ${ }^{11}$ In 27 malnourished patients, 3 days of continuous infusion followed by cyclic infusion significantly improved nitrogen balance. ${ }^{12}$ Two studies using this technique also demonstrated increases in serum albumin levels $(\mathrm{N}=14,27$, respectively). ${ }^{8,12}$ However, a randomized controlled trial of patients with a history of hepatobiliary dysfunction showed no differences in serum albumin levels with cyclic infusion compared with continuous infusion $(\mathrm{N}=65) \cdot{ }^{13}$ Four patients with corticosteroid-induced hypercatabolism who switched to cyclic PN infusion following a period of continuous infusion had lower nitrogen balance with cyclic infusion in 1 study. ${ }^{9}$

Two randomized parallel-group studies of sequential bolus infusion, a method in which nutrition is supplied in a series of six 1-hour infusions daily, showed higher nitrogen balance compared with continuous infusion in patients following cholecystectomy $(\mathrm{N}=30)^{14}$ but lower nitrogen balance in patients following major surgery $(\mathrm{N}=65){ }^{15}$
Carbohydrates, fat, and respiratory quotient. Two beforeafter studies showed higher blood glucose concentrations during the infusion period compared with the noninfusion period of cyclic $\mathrm{PN}$ regimens, with a quick return to baseline values following discontinuation of PN infusion ( $\mathbf{N}=15,4$, respectively). ${ }^{16,17}$ Two studies, one of which was a randomized crossover study, demonstrated similar rates of carbohydrate $(\mathrm{CHO})$ and fat oxidation between cyclic and continuous PN infusions during infusion per nonprotein respiratory quotient $(\mathrm{RQ} ; \mathrm{N}=5,8){ }^{6,10}$ During the postinfusion period of cyclic $\mathrm{PN}$, CHO oxidation decreased and fat oxidation increased. ${ }^{6,10}$ There was net lipogenesis and nonprotein $\mathrm{RQ}>1$ during infusion, compared with net lipolysis and nonprotein $\mathrm{RQ}<1$ following discontinuation. ${ }^{6,10}$ Hepatic triglyceride secretion was not affected by infusion schedule in a randomized parallelgroup study $(\mathrm{N}=22)^{18}$ nor was nighttime oxygen consumption in a small nonrandomized parallel-group study $(\mathrm{N}=8) .{ }^{19}$ A randomized parallel-group study of 16 traumatized or infected patients requiring mechanical ventilation demonstrated greater increases in $\mathrm{O}_{2}$ uptake and $\mathrm{CO}_{2}$ elimination with initiation of cyclic $\mathrm{PN}$ infusion compared with continuous infusion. ${ }^{11}$ Changes in RQ with PN were similar between groups.

Energy expenditure. In 3 studies, of which 2 used a randomized crossover design, daily energy expenditure was similar with cyclic and continuous PN infusion $(\mathrm{N}=$ 5-12). ${ }^{6,7,10}$ Energy expenditure increased during infusion of cyclic PN regimens and decreased during the noninfusion period, whereas it remained relatively constant throughout the day with continuous infusion. ${ }^{6,7,10}$ Sequential bolus infusion resulted in lower overall energy balance following cholecystectomy in a randomized parallel-group study $(\mathrm{N}=30) .{ }^{14}$ In a randomized parallelgroup study of 16 traumatized or infected patients requiring mechanical ventilation, energy expenditure increased when patients began cyclic or continuous PN infusions; however, the increases were greater in the cyclic vs continuous infusion group. ${ }^{11}$ These greater increases in energy expenditure were accompanied by greater thermogenic and body temperature responses but similar heart rates and blood pressures.

Hormones, catecholamines, and circadian rhythms. A number of before-after studies demonstrated a rapid increase in serum insulin concentrations upon PN infusion initiation, with a rapid decrease following discontinuation $(\mathrm{N}=$ 4-48). 5,16,17,20 Two of these studies demonstrated similar circulating glucagon, cortisol, and growth hormone concentrations between patients receiving cyclic and continuous $\mathrm{PN}$ infusion $\left(\mathbf{N}=10,48\right.$, respectively). ${ }^{5,20}$ In a randomized parallel-group study of 16 traumatized or 
infected mechanically ventilated patients, urinary epinephrine, norepinephrine, and cortisol excretion rates were similar between patients given cyclic and continuous infusion, although circulating cortisol concentrations were elevated relative to baseline during infusion of cyclic regimens. ${ }^{11}$ One small study demonstrated circadian rhythms of serum levels of total proteins, total cholesterol, high- and low-density lipoproteins, triglycerides, free fatty acids, and apolipoproteins A and B during cyclic PN $(\mathrm{N}=6) .^{21}$

\section{Hepatic Complications}

Adults. One randomized parallel-group study of patients with a history of hepatobiliary dysfunction during PN treatment showed stabilization of serum levels of total and direct bilirubin, alkaline phosphatase, serum glutamic oxaloacetic transaminase (SGOT), and serum glutamic pyruvic transaminase (SGPT) if cyclic PN infusion was initiated when total serum bilirubin was $<20 \mathrm{mg} / \mathrm{dL}$ ( $\mathrm{N}=$ 65). ${ }^{13}$ Among patients randomly assigned to receive continuous infusion, patients with baseline serum total bilirubin levels of $5-10 \mathrm{mg} / \mathrm{dL}$ developed increased serum levels of total bilirubin, direct bilirubin, and alkaline phosphatase; patients with baseline serum total bilirubin levels of $10-20 \mathrm{mg} / \mathrm{dL}$ developed increased serum levels of total bilirubin, direct bilirubin, SGOT, and SGPT. No benefit of cyclic PN infusion was seen in patients with baseline serum total bilirubin levels $>20 \mathrm{mg} / \mathrm{dL}$. A study in which 14 patients were switched to cyclic PN infusion following a period of continuous infusion demonstrated significant decreases in serum levels of alkaline phosphatase and lactate dehydrogenase with the cyclic regimen and nonsignificant decreases in serum levels of bilirubin and SGOT. ${ }^{8}$ These changes were anecdotally noted to occur with remittance of hepatomegaly and decreases in hepatic infiltration of glycogen and fat seen on serial biopsy.

Children. One study of 10 infants stabilized on continuous PN infusion, and subsequently switched to cyclic infusion, showed an increase in serum bilirubin levels in the 2 weeks prior to cycling, with a nonsignificant decease in the 2 weeks following cycling initiation..$^{22}$ In a study of 10 neonates receiving PN as a series of three 4-hour infusions daily, no patients experienced elevations in serum SGOT or SGPT levels during treatment, and 1 patient had elevation of direct bilirubin; 18\% of historical controls developed cholestatic jaundice, $61 \%$ developed elevated serum SGOT, and $46 \%$ developed elevated serum SGPT. ${ }^{23}$

\section{Glycemic Control}

Adults. Abrupt initiation of PN infusion was shown to cause blood glucose elevation, primarily in the first 60 minutes of infusion, in a before-after study $(\mathrm{N}=18){ }^{24}$ Four studies examining the safety of abruptly discontinuing PN infusion in adults showed transient decreases in blood glucose, primarily in the first hour following infusion discontinuation, without symptomatic hypoglycemia $(\mathrm{N}=12-48){ }^{20,24-26}$ Based on the results of a randomized crossover study, abrupt discontinuation and tapered discontinuation of PN infusion are associated with similar responses in blood insulin, glucagon, epinephrine, norepinephrine, cortisol, and growth hormone in the first 90 minutes following discontinuation $(\mathrm{N}=12) .{ }^{25}$

Children. Abrupt PN discontinuation in 15 children 2.411.4 years old led to no symptomatic hypoglycemia in a before-after study. ${ }^{16}$ A randomized crossover study of 10 patients 1.5-36 months old showed significantly greater decreases in blood glucose levels with abrupt discontinuation compared with tapered discontinuation and higher rates of hypoglycemia ( $55 \%$ vs $20 \%, P$ value not reported). ${ }^{27}$

\section{Metabolic Bone Disease}

Two studies demonstrated increased urinary calcium, magnesium, and phosphorus excretion during cyclic PN infusion, in 1 case relative to the noninfusion period $(\mathbf{N}=16)^{28}$ and, in the other, relative to excretion levels during continuous infusion (as measured in a historical cohort, $\mathrm{N}=10) .{ }^{29}$ However, no differences between cyclic and continuous infusion in 24-hour renal filtered calcium load, fractional calcium excretion, or blood concentrations of calcium, phosphorus, magnesium, or 25-hydroxy vitamin $\mathrm{D}$ were observed. ${ }^{29}$

\section{Discussion}

A total of 25 clinical studies met inclusion criteria for review. Sample sizes ranged from 4 to 65 , with 18 of 25 studies examining $\leq 20$ patients and 9 of 25 studies examining $\leq 10$ patients. Ten studies used randomized designs, and several others appropriately applied a before-after design to investigate short-term metabolic changes in individuals upon initiation or discontinuation of infusion. In 4 studies, changes in metabolic parameters were reported in the context of a before-after design in which an earlier continuous infusion regimen was transitioned to a later cyclic infusion regimen. ${ }^{8,12,17,22}$ Although patients were stabilized to varying degrees prior to initiation of cyclic infusion in these studies, the course of disease over time could confound the relationship between infusion regimen and metabolic parameter, drawing into question any inferences about the impact of infusion schedule. In other studies, comparison to a historical control, ${ }^{23,29}$ use of nonrandomized treatment assignments, ${ }^{10,19}$ and pooling of patients receiving sequential infusion regimens 
with cyclic infusion patients ${ }^{8}$ had a similar impact on interpretability. Fortunately, none of these studies has major treatment implications in the absence of further confirmatory data.

Cyclic and continuous PN infusions produce similar nitrogen balance in most patients. Sequential bolus infusion of PN produced improved nitrogen balance in an initial study, but the technique has not been widely researched or adopted in clinical practice. Carbohydrate and lipid metabolism are similar with cyclic or continuous infusion during infusion. During the postinfusion period of cyclic PN regimens, carbohydrate metabolism decreases, fat metabolism increases, and $R Q$ decreases. Nonprotein RQ decreases between the infusion and noninfusion states, generally from $>1$ to $<1$. In traumatized or infected mechanically ventilated patients, cyclic infusion may be disadvantageous because of an increase in carbon dioxide production. Circulating concentrations of counterregulatory hormones are similar with cyclic and continuous PN infusion regimens.

Patients with hyperbilirubinemia receiving continuous PN infusion may experience stabilization in liver function tests with a switch to cyclic PN infusion. This benefit may not extend to patients with precycling serum bilirubin levels of $\geq 20 \mathrm{mg} / \mathrm{dL}$. Further prospective evidence of benefit from cyclic PN infusion with respect to hepatobiliary complications in adults is anecdotal. One prospective study in infants who were switched from continuous to cyclic PN infusion demonstrated stabilization and possible decreases in serum bilirubin levels with initiation of cyclic PN infusion.

Abrupt initiation of cyclic PN infusion may lead to transient hyperglycemia in some patients. Abrupt discontinuation of cyclic PN infusion in adults has not been associated with symptomatic hypoglycemia and has shown no impact on hormone and catecholamine responses to infusion termination. In children, abrupt discontinuation of PN infusion is most likely to cause hypoglycemia in patients younger than 2-3 years old, whereas older children have shown similar results to adults.

Although data are limited, cyclic PN infusion has not been associated with increased daily losses of calcium, phosphorus, magnesium, or vitamin D compared with continuous infusion. Although calcium loss increases during cyclic PN infusion, there is little evidence that this could contribute to metabolic bone disease.

\section{Conclusion}

Clinical data support the current widespread use of cyclic PN infusion in most populations. Although practicality is the primary impetus for cyclic PN infusion and likely its greatest benefit, clinical studies have played an important role in confirming that the modality is generally not metabolically disadvantageous. Some benefit may be realized in prevention of hepatobiliary disease, but some precautions must be taken to avoid hypoglycemia, particularly in young children. Acutely ill mechanically ventilated patients may experience increased carbon dioxide production with cyclic PN infusion and therefore may derive greater benefit from continuous infusion.

\section{References}

1. A.S.P.E.N. Board of Directors and the Clinical Guidelines Task Force. Guidelines for the use of parenteral and enteral nutrition in adult and pediatric patients [erata in JPEN 2002;26:144]. JPEN J Parenter Enteral Nutr. 2002;26(1 suppl):1SA-138SA.

2. Btaiche IF, Khalidi N. Parenteral nutrition-associated liver complications in children. Pharmacotherapy. 2002;22:188-211.

3. Btaiche IF, Khalidi N. Metabolic complications of parenteral nutrition in adults, part 2. Am J Health Syst Pharm. 2004;61:2050-2057.

4. Btaiche IF, Khalidi N. Metabolic complications of parenteral nutrition in adults, part 1. Am J Health Syst Pharm. 2004;61:1938-1949.

5. Byrne WJ, Lippe BM, Strobel CT, Levin SR, Ament ME, Kaplan SA. Adaptation to increasing loads of total parenteral nutrition: metabolic, endocrine, and insulin receptor responses. Gastroenterology. 1981; 80(5 pt 1):947-956.

6. Just B, Messing B, Darmaun D, Rongier M, Camillo E. Comparison of substrate utilization by indirect calorimetry during cyclic and continuous total parenteral nutrition. Am J Clin Nutr. 1990;51:107-111.

7. Lerebours E, Rimbert A, Hecketsweiler B, Hellot MF, Denis P, Colin R. Comparison of the effects of continuous and cyclic nocturnal parenteral nutrition on energy expenditure and protein metabolism. JPEN J Parenter Enteral Nutr. 1988;12:360-364.

8. Maini B, Blackburn GL, Bistrian BR, et al. Cyclic hyperalimentation: an optimal technique for preservation of visceral protein. $J$ Surg Res. 1976;20:515-525.

9. Messing B, Pontal PJ, Bernier JJ. Metabolic study during cyclic total parenteral nutrition in adult patients with and without corticosteroidinduced hypercatabolism: comparison with standard total parenteral nutrition. JPEN J Parenter Enteral Nutr. 1983;7:21-25.

10. Pullicino E, Goldberg GR, Elia M. Energy expenditure and substrate metabolism measured by $24 \mathrm{~h}$ whole-body calorimetry in patients receiving cyclic and continuous total parenteral nutrition. Clin Sci (Lond). 1991;80:571-582.

11. Forsberg E, Soop M, Lepapea A, ThörneA. Metabolic and thermogenic response to continuous and cyclic total parenteral nutrition in traumatised and infected patients. Clin Nutr. 1994;13:291-301.

12. Matuchansky C, Morichau-Beauchant M, Druart F, Tapin J. Cyclic (nocturnal) total parenteral nutrition in hospitalized adult patients with severe digestive diseases: report of a prospective study. Gastroenterology. 1981;81:433-437.

13. Hwang TL, Lue MC, Chen LL. Early use of cyclic TPN prevents further deterioration of liver functions for the TPN patients with impaired liver function. Hepatogastroenterology. 2000;47:1347-1350.

14. Hyltander A, Arfvidsson B, Körner U, Sandström R, Lundholm K. Metabolic rate and nitrogen balance in patients receiving bolus intermittent total parenteral nutrition infusion. JPEN J Parenter Enteral Nutr. 1993;17:158-164.

15. Sandström R, Hyltander A, Körner U, Lundholm K. The effect on energy and nitrogen metabolism by continuous, bolus, or sequential infusion of a defined total parenteral nutrition formulation in patients after major surgical procedures. JPEN J Parenter Enteral Nutr. 1995;19:333-340. 
16. Werlin SL, Wyatt D, Camitta B. Effect of abrupt discontinuation of high glucose infusion rates during parenteral nutrition. $J$ Pediatr. 1994;124:441-444.

17. Wood RJ, Bengoa JM, Rosenberg IH. Urinary C-peptide measurements in patients receiving continuous and cyclic total parenteral nutrition. J Lab Clin Med. 1985;105:259-264.

18. Isabel-Martinez L, Skinner C, Parkin A, Hall R. Comparison of the effects of enteral feeding with continuous and intermittent parenteral nutrition on hepatic triglyceride secretion in human beings. Surgery. 1989;105:415-420.

19. Fagioli I, Bes F, Franc B, Putet G, Ricour C, Salzarulo P. Oxygen consumption during sleep in children under continuous and cyclic nutrition. Physiol Behav. 1991:49:1159-1162.

20. Wagman LD, Newsome HH, Miller KB, Thomas RB, Weir GC. The effect of acute discontinuation of total parenteral nutrition. Ann Surg. 1986;204:524-529.

21. Matuchansky C, Fabre J, Guillard O, Morichau-Beauchant M, Reinberg A. Effects of cyclic (nocturnal) total parenteral nutrition and continuous enteral nutrition on circadian rhythms of blood lipids, lipoproteins, and apolipoproteins in humans. Am J Clin Nutr. 1985:41:727-734.

22. Collier S, Crough J, Hendricks K, Caballero B. Use of cyclic parenteral nutrition in infants less than 6 months of age. Nutr Clin Pract. 1994;9:65-68.
23. Takehara H, Hino M, Kameoka K, Komi N. A new method of total parenteral nutrition for surgical neonates: it is possible that cyclic TPN prevents intrahepatic cholestasis. Tokushima Exp Med. 1990;37:97-102.

24. Krzywda EA, Andris DA, Whipple JK, et al. Glucose response to abrupt initiation and discontinuation of total parenteral nutrition. JPEN J Parenter Enteral Nutr. 1993;17:64-67.

25. Eisenberg PG, Gianino S, Clutter WE, Fleshman JW. Abrupt discontinuation of cycled parenteral nutrition is safe. Dis Colon Rectum. 1995;38:933-939.

26. Nirula R, Yamada K, Waxman K. The effect of abrupt cessation of total parenteral nutrition on serum glucose: a randomized trial. Am Surg. 2000;66:866-869.

27. Bendorf K, Friesen CA, Roberts CC. Glucose response to discontinuation of parenteral nutrition in patients less than 3 years of age. JPEN J Parenter Enteral Nutr. 1996;20:120-122.

28. Boncompain-Gérard M, Robert D, Fouque D, Hadj-Aïssa A. Renal function and urinary excretion of electrolytes in patients receiving cyclic parenteral nutrition. JPEN J Parenter Enteral Nutr. 2000;24:234-239.

29. Wood RJ, Bengoa JM, Sitrin MD, Rosenberg IH. Calciuretic effect of cyclic versus continuous total parenteral nutrition. Am J Clin Nutr. 1985;41:614-619. 